\title{
Geschichten über Lucifer im anonymen Meistergesang des fünfzehnten und sechzehnten Jahrhunderts. Unter besonderer Berücksichtigung der Lieder in Regenbogens ,Langem Ton“
}

\section{Begriffsgeschichtliche Hinführung}

Teufels- oder Satansdarstellungen sind im abendländischen Mittelalter sowohl in der Kunst als auch in der Literatur weit verbreitet und weisen auf ein reges, bis heute ungebrochenes Interesse am Bösen und dessen Verkörperungen. ${ }^{1}$ Ihren Ursprung haben die durchaus facettenreichen Darstellungen der Figur des Teufels sowohl im Alten als auch im Neuen Testament (wobei sich zwischen den beiden Bibelteilen ein noch näher zu erläuternder Wandel in der Beschreibung vollzieht) sowie maßgeblich in der Bibelauslegung durch die Kirchenväter.

Verwirrende Vielfalt herrscht bezüglich der Terminologie: Das Alte Testament nutzt mehrfach das Lexem satan, doch die Bezeichnung ,Satan“ ergibt hinsichtlich ihrer Herkunft und der Beschreibung der Funktion des Bezeichneten kein einheitliches Bild. ${ }^{2}$ Das Neue Testament verwendet sowohl satan als auch diabolos, woraus sich über das mittellateinische diuvalus, das althochdeutsche tiuvel und das mittelhochdeutsche tiufel, tievel unser heutiges Wort ,Teufel' entwickelte. Dennoch sind Teufel und Satan nicht ohne weiteres gleichzusetzen, denn der hebräische Ausdruck satan, der im Alten Testament sowohl als Verb wie auch als Nomen vorkommt, bezeichnet ganz generell die Rollen von Gegenspielern; ${ }^{3}$ so

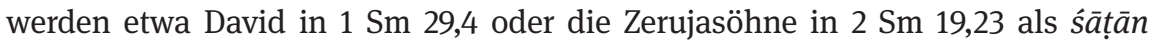
bezeichnet. Darüber hinaus kann die Benennung als Satan aber auch ermögli-

1 Besonders intensiv mit dem Thema befasst haben sich Wehrle, Pagels, Flasch, Martinek und Schmidt, auf deren Arbeiten die folgende Hinführung beruht.

2 Vgl. Wehrle, S. 194.

3 Vgl. ebd. Wehrle setzt sich ausführlich mit den sprachlichen Befunden zum Nomen śățān (27 Belege) sowie zum gleichlautenden, wohl denominierten Verb (6 Belege) im Alten Testament auseinander. Vgl. hierzu auch die Ausführungen von Day, S. 25-43. 
chen, konkrete Feinde als Verkörperungen transzendenter Mächte auszumachen und auf eine himmlische oder dämonische Sphäre zu verweisen. ${ }^{4}$

,Satan` ist somit zunächst einmal nicht der Name einer individuellen Figur. Dennoch gibt es auch im Alten Testament zwei Stellen, an denen Satan als Gestalt mit fest umrissenen Aufgaben und als Ankläger der Menschen vor Gott in Erscheinung tritt: ${ }^{5} \mathrm{im}$ Buch Hiob und bei Sacharja. Während Satan bei Sacharja eine eher unbedeutende Rolle spielt - er kommt gar nicht dazu, Josua zu verklagen, denn Gottvater fährt ihm ziemlich rüde über den Mund $-{ }^{6}{ }^{6}$ ist das Buch Hiob die einzige alttestamentarische Schrift, in der Satan eine entscheidende Rolle zukommt. Auch hier ist Satan allerdings kein Gegenspieler Gottes, sondern einer der ,Gottessöhne', die zu Gottes Hofstaat gehören und sich vor Gott zu versammeln pflegen. ${ }^{7}$ Er wird als Skeptiker beschrieben, der vom Menschen nichts Gutes erwartet und so die Gottesfurcht Hiobs nur auf dessen Wohlergehen zurückführt. ${ }^{8}$ Die Heimsuchungen Hiobs erfolgen ausschließlich mit Gottes Zustimmung; die von Gott gesetzten Grenzen überschreitet Satan nicht. Zum Widersacher Gottes wird Satan erst im Neuen Testament.

In dem Maße, in dem Satan zu einer immer bedeutsameren und stärker als Person umrissenen Figur wurde, begannen die Geschichten über seinen Ursprung zu wuchern. Für die spätere Gleichsetzung von Lucifer mit Satan, die auch im Meistergesang immer wieder vollzogen wird, ist eine Passage aus dem Buch des Propheten Jesaja besonders wichtig:

quomodo cecidisti de caelo lucifer qui mane oriebaris corruisti in terram qui vulnerabas gentes qui dicebas in corde tuo in caelum conscendam super astra Dei exaltabo solium meum sedebo in monte testamenti in lateribus aquilonis ascendam super altitudinem nu-

4 Vgl. Pagels, S. 39. Vgl. auch S. 73: „Wenn die hebräischen Erzähler schon im sechsten Jahrhundert vor unserer Zeitrechnung gelegentlich eine übernatürliche Figur einführen, die sie als Satan bezeichneten, so meinten sie damit nur irgendeinen der Engel, die Gott zu dem speziellen Zweck geschickt hatte, menschliches Tun zu behindern oder zu vereiteln“.

5 Vgl. Flasch, S. 75: „Die griechischen Übersetzer des Alten Testaments, die angeblich zu siebzigst um 200 v.Chr. die sog. Septuaginta schufen, nannten ihn [d.i. der Teufel, Anm. JLa] ho diabolos, nicht mehr nur diabolos, also „Feind“ oder ,Ankläger‘ allgemein, auch auf Menschen bezogen, sondern als den Teufel mit bestimmtem Artikel.“

6 Za 3,1f.: et ostendit mihi Iesum sacerdotem magnum stantem coram angelo Domini et Satan stabat a dextris eius ut adversaretur ei et dixit Dominus ad Satan increpet Dominus in te Satan et increpet Dominus in te qui elegit Hierusalem numquid non iste torris est erutus de igne.

7 Vgl. zur Frage, um wen es sich bei den sog. Göttersöhnen handelt Flasch, S. 75, sowie Pagels, S. 75 .

8 Day, S. 80 f., sieht mit Berufung auf David Robertson, S. 451, und Edwin Good, S. 481, nicht die Gottesfurcht Hiobs auf den Prüfstand gestellt, sondern „that Yahweh is on trial for his conduct of world order, from the very beginning.“ 
bium ero similis Altissimo verumtamen ad infernum detraheris in profundum laci [Is $14,12-15]^{9}$

Tatsächlich bezieht sich der Text der Prophezeiung auf einen nicht identifizierten babylonischen König. Dabei gebraucht er das Bild vom gefallenen Morgenstern (lucifer), ${ }^{10}$ ohne jedoch schon auf einen wohl noch nicht etablierten Mythos von der Rebellion des Engels anzuspielen. ${ }^{11}$ Erst im Neuen Testament wird, wohl zurückgehend auf eine vorbiblische jüdische Apokryphe, ${ }^{12}$ das Bild bei Lc 10,18 auf Satan bezogen: „Ich sah den Satan vom Himmel fallen wie einen Blitz“ (Lutherbibel 2017). Aber den wohl größten Einfluss auf die zahlreich folgenden, teils phantastisch anmutenden Aufbereitungen der Geschichte bewirkte wohl die christliche Nacherzählung seines Himmelssturzes in der stark durch gnostische Einflüsse geprägten Offenbarung des Johannes, in der der vom Himmel stürzende Satan allerdings durch einen Drachen ersetzt wird (vgl. Apc 12). Satan wird hier zur Schlange und zum Anführer der gefallenen Engel. Deshalb deuteten die Kirchenväter die verhängnisvolle Unterhaltung im Paradies als Gespräch Evas mit dem Teufel; diese Deutung entstand im ersten nachchristlichen Jahrhundert und verschob die Rolle Satans maßgeblich vom Ankläger im Alten Testament zum Versucher. ${ }^{13}$ Die Annahme der Kirchenväter, es handele sich bei Satan um eine überzeitliche Realität, erlaubte es späteren Generationen, ihn im Gegensatz zum

9 Zitiert nach der Vulgata. Deutsche Übersetzung gemäß der Lutherbibel 2017: „Wie bist du vom Himmel gefallen, du schöner Morgenstern! Wie wurdest du zu Boden geschlagen, du Bezwinger der Völker! Du aber gedachtest in deinem Herzen: ,Ich will in den Himmel steigen und meinen Thron über die Sterne Gottes erhöhen, ich will mich setzen auf den Berg der Versammlung im fernsten Norden. Ich will auffahren über die hohen Wolken und gleich sein dem Allerhöchsten. Doch hinunter ins Totenreich fährst du, in die tiefste Grube!“

10 Es ist, so Schmidt, anzunehmen, dass sich die Bibelstelle auf eine frühe (Fehl)deutung des griechischen Phäethon-Mythos bezieht. Dieser berichtet davon, wie Phäethon, der Sohn des Helios, seinen Vater überredet, dessen Sonnenwagen einmal lenken zu dürfen. Dies überfordert den Sohn jedoch, sodass er aus der Bahn gerät und schließlich von Zeus mit einem Blitz gestoppt werden muss. Dabei stürzt Phäethon vom Himmel. Jesaja vergleicht also ,jenen nichtidentifizierten babylonischen König mit Eosphoros [d.h. einer Personifikation des Morgensterns Venus, Anm. JLa], auf den er die offenbar verfälschte Geschichte des Phäeton bezieht“ (Schmidt, S. 21). 11 Vgl. ebd.

12 Nicht nur Lukas, sondern alle vier Evangelisten ,verbinden die biographische Form mit der Thematisierung übernatürlicher Konflikte, die sie der jüdischen apokalyptischen Literatur entlehnen, um eine neue Erzählform zu schaffen“ (Pagels, S. 39).

13 In dieser Beschreibung innerhalb der Offenbarung des Johannes liegt die Tatsache begründet, dass etwa die Schlange, die im Garten Eden Eva verführt, noch heute mit Satan identifiziert wird. Nicht nur das Lukasevangelium, sondern auch die drei übrigen Evangelisten sind darauf bedacht zu zeigen, was die geschilderten Ereignisse eschatologisch bedeuten. 
Genesistext auch zum Urheber des Sündenfalls zu erheben. ${ }^{14}$ Die dem Teufel zugeschriebene Macht und Bedeutung steigern sich im Laufe des zweiten Jahrhunderts zunehmend. ${ }^{15}$ Zieht man die übrigen Stellen, an denen die Bibel Satan nennt und sein Handeln thematisiert, heran, wird ein Wandel vom Alten zum Neuen Testament erkennbar. Satan wird vom Ankläger der Menschheit (siehe Hiob, Sacharja, Numeri) zum Feind Gottes und Christi im Neuen Testament (vgl. z. B. 2 Cor 4,4 u. Mc 1,13), wo er als eine den Menschen hindernde (vgl. z. B. 1 Th 2,18) und sein Heil gefährdende Macht (vgl. z. B. Lc 22,31 u. 2 Cor 11,3) erscheint. Satan wird neben der Paradiesesschlange (Apc 12,9 u. 20,2) auch mit Judas (vgl. Io 6,70) und mit Petrus (vgl. Mc 8, 33) identifiziert, der Jesus davon abhalten will, seinen Weg zu Ende zu gehen. Wo immer Satan im Neuen Testament in Erscheinung tritt, versucht er, den Heilsplan Christi zu verhindern, wobei er sowohl gegen Jesus als auch gegen dessen Lehre intrigiert. Allerdings ist auch hierbei zu bemerken, dass Satan nach der gängigen Auffassung der Kirchenlehrer nicht unabhängig von Gott handeln kann. ${ }^{16}$

Von Jesaja, Lukas und der Offenbarung des Johannes ausgehend, identifiziert zunächst Origenes Lucifer mit dem Satan. ${ }^{17}$ Nach Origenes’ Auffassung war Stolz die Ursache des Falls der Engel und ihrer Absicht, ihren Willen über den Willen

14 Die Annahme einer überzeitlichen Realität des Teufels (und damit eine Gleichsetzung von Paradiesschlange und Teufel) findet sich bis ins neunzehnte Jahrhundert auch unter Dogmatikern. Vgl. etwa Scheeben, S. 591. Kurt Flasch, S. 74, betont in seiner Studie hingegen die „historische Existenz"Satans und versucht, die Figur und ihren Wandel chronologisch durch die Zeit zu verfolgen.

15 Vgl. Flasch, S. 73, der zudem herausstellt, dass sich Satansbild und Gottesbild in gleichem Maße ändern. Agiert Gott in den frühen Texten mitunter noch mit „archaischer Rohheit“, wird das mit dieser Rohheit verbundene Schlechte und Böse in der Welt sukzessive dem Teufel zugeschrieben: „Satan ist entstanden, als Jahweh sich moralisch abqualifizierte. Im achten Jahrhundert konnte Jahweh noch von sich sagen: ,Es geschieht kein Unglück in irgendeiner Stadt, ohne daß der Herr es bewirkt hat', Anos 3, 6. Noch zwei Jahrhunderte später rühmte er sich: „Ich erschaffe das Licht und mache das Dunkel. Ich bewirke das Heil und erschaffe das Unheil. Deuterojesaja 45, 7. [...] Es bedurfte nicht des Teufels, um das Schlechte in Gottes Welt plausibel zu machen." Ebd., S. 76.

16 So lehnt etwa Augustinus die als häretisch geltende manichäische Lehre vom Dualismus von Satan und Gott ab. Er befasst sich ausführlich mit der Frage, ob Satan bereits von seinem bevorstehenden Fall wusste und ob er in dieser Hinsicht jemals eine Wahl hatte. In diesem $\mathrm{Zu}$ sammenhang unterscheidet Augustinus zwei Arten von Engeln: die über den Himmeln lebenden Wesen einerseits, die in ewigem Segen leben, und andererseits die weltlichere Sorte, die auf diesen Segen hoffen kann, wenn sie den Geboten Gottes Folge leistet. Den Satan zählt er zur zweiten Sorte, kann jedoch in der Bibel keinen Beleg für seine These finden. Vgl. Rees, S. 262f. Zum iranischen/persischen Dualismus, der den spätjüdischen und frühchristlichen Satansglauben maßgeblich beeinflusste, vgl. etwa Schmidt, S. 22-24.

17 In der Nachfolge des Origenes stehen u. a. Eusebius, Tertullian und Papst Gregor I. 
Gottes zu stellen. ${ }^{18}$ Origenes positioniert sich somit klar in der Frage, ob Lucifer bereits böse geschaffen wurde und aus welchen Gründen es zu seinem Sturz kam. Es erscheint ihm unmöglich, dass Teufel und Dämonen von einem ausnahmslos guten Gott als böse Wesen geschaffen wurden. Den Grund für den Sturz Lucifers sieht er als in ihm selbst, in seiner Existenz als freiem Wesen begründet. ${ }^{19}$ Kurt Flasch spricht in Bezugnahme auf Origenes von „antigöttlichen Ambitionen“, ${ }^{20}$ aufgrund derer Satan böse geworden sei. Zur Bekräftigung seiner Argumentation verbindet Origenes seine Überlegungen mit der genannten Stelle aus dem Buch Jesaja. Den dort genannten Lucifer verbindet der Kirchenvater zwar noch mit dem König von Tyrus aus Ez 28,12, seine Ausführungen zeigen jedoch,

dass es sich bei dem angesprochenen König nicht um einen Menschen handeln kann, sondern, ,daß diese Macht vorher heilig und selig war und aus dieser Seligkeit, nachdem in ihr Missetat gefunden wurde, niederstürzte zur Erde und daß sie nicht von Natur und durch die Schöpfung von solcher Art gewesen ist.$^{21}$

Diese Verbindung von biblischer Tradition und christlich-exegetischer Interpretation bei Origenes legte den Grundstein für eine reichhaltige Legendenbildung um Lucifer. ${ }^{22}$ Dabei ist nachträglich nicht zu klären, ob diese Gleichsetzung irrtümlich geschah oder im Hinblick auf einen apologetischen Gedanken absichtsvoll vollzogen wurde. In jedem Fall sollte die daraus resultierende Komplexität des Teufelsbildes „für die Geschichte der Satansvorstellung von entscheidender Bedeutung sein“. ${ }^{23}$ In dem Augenblick, in dem das primär namen- und gestaltlose Böse, das unter der Bezeichnung Satan in unterschiedlichen Rollen aufzutreten vermag, sowohl einen Eigennamen als auch eine Geschichte erhält, eröffnen sich neue Perspektiven im Umgang mit diesem als personalisierte Projektionsfläche. Satan wird im Sinne des Neuen Testaments verstärkt als Antagonist Gottes dargestellt, der das Böse in der Welt verkörpert und die Menschen versucht.

18 Keine Rolle spielten für Origenes die Aspekte Neid und Begierde, da er den Fall Satans und seiner Begleiter nicht mit der Erschaffung der Menschen zusammenbringt. Sehr wohl deutet Origenes den Fall Lucifers als dessen eigenen Fehler und nicht als Teil des göttlichen Plans. Vgl. Martinek, S. 150.

19 Vgl. Origenes, I, 5, 2-5, S. 195-213 und I, 6, 1-4, S. 214-131.

20 Flasch, S. 92 und vgl. ausführlich zur Bedeutung des Origenes für den Teufelsglauben ebd., S. $89-94$.

21 Lies, S. 72 mit Zitat aus PA I 5,4.

22 Vgl. Martinek, S. 151.

23 Schmidt, S. 21. 
Vom theologischen Denken der Kirchenväter bestimmt, findet der Teufelsglaube in der laikalen Frömmigkeit ihren fruchtbarsten Boden. ${ }^{24}$ Auch in den Meistergesang des fünfzehnten und sechszehnten Jahrhunderts findet Satan Eingang, wobei gerade in dieser, der Vermittlung von (religiösem) Wissen verpflichteten Kunstform eine explizite Unterscheidung der Begrifflichkeiten Teufel und Satan sowie dem Eigenamen Lucifer vorgenommen zu sein scheint. Dass die Meistersinger schon mit dem ersten Aufkommen des meisterlichen Liedes ${ }^{25}$ einen geistlichen, explizit ,laientheologischen“ Anspruch an ihre Lieder stellten, hält bereits Karl Stackmann im Nachwort seiner Heinrich von Mügeln-Ausgabe aus dem Jahr 1958 fest. ${ }^{26}$ Dieser Anspruch verstärke sich in der Entwicklung von der Sangspruchdichtung bis zum institutionellen Meistergesang mit der zunehmenden Konzentration auf geistliche Themen, bis er schlussendlich in die Reformation münde und in Form von Bibeldichtung zum zentralen Movens des Meistergesangs werde. ${ }^{27}$ Spätestens mit der Institutionalisierung des Meistergesangs in den großen Meistersingergesellschaften und der damit einhergehenden öffentlichen Kunstausübung in sogenannten Singschulen wird die meisterliche Lied-

24 Neben der Vorstellung vom Fegefeuer entwickelt sich im zwölften Jahrhundert auch die Geographie der Hölle weiter, in der die Sünder nach der Schwere ihrer Schuld beurteilt und unterschiedlich bestraft werden. Berichte über visionäre Reisen ins Jenseits, in denen sowohl die Strukturen als auch der Aufbau des Jenseits verhandelt werden, erreichen in diesem Zeitraum ihren Höhepunkt, nachdem sie sich bereits im Frühmittelalter als eigenständige Gattung etablieren konnten. Schon in der Bibel vertreten, findet das Thema der Verführung des Menschen durch den Teufel eine große Resonanz und verbreitet sich mittels früher Geschichtsdarstellungen (z.B. Radulf Glabers Historiae, Guibert von Nogents autobiographische Erzählung De vita sua u.a.) Predigten und Exempelsammlungen (z. B. Richalm von Schöntal oder Caesarius von Heisterbach). Vgl. zur Entwicklung der literarischen Jenseitsvisionen von der Spätantike bis Dante Dinzelbacher, S. 64-72.

25 Das meisterliche Lied ist Teil eines Gattungskontinuums, welches sich vom Sangspruch des dreizehnten Jahrhunderts bis zum Meistergesang des siebzehnten und achtzehnten Jahrhunderts erstreckt. Der Terminus ,meisterliche Liedkunst‘ wurde von Frieder Schanze eingeführt, um die hinsichtlich der institutionellen Gebrauchssituation der Lieder und dem sozialen Status der Autoren schwer fassbare Übergangsphase zwischen „nicht mehr Sangspruchdichtung und noch nicht ausschließlich Meistergesang“ (Schanze, S. 10) zu bezeichnen.

26 Vgl. Heinrich von Mügeln, S. 179. Rosmer (S. 310) betont eine Dominanz geistlicher Themen in den frühen Meisterlied-Sammlungen des 15. Jahrhunderts, die sich im Textkonvolut der bis zur Mitte des 14. Jahrhunderts überlieferten Sangsprüche noch nicht feststellen lässt.

27 Ebd., S. 177. Der schon von Stackmann (ebd., S. 180) aufgeworfenen Frage, inwieweit die meister des vierzehnten und fünfzehnten Jahrhunderts „durch ihre kunst, deren Resultat letzten Endes in der Erwerbung des Mitspracherechts von Laien in theologischen Fragen bestand, der Reformation an einem entscheidenden Punkt den Weg geebnet haben“, widmet sich jüngst Felix Prautzsch in einem noch nicht veröffentlichten Beitrag zum ästhetischen und theologischen Anspruch im Meistergesang. 
kunst zu einer Kunstübung, der es maßgeblich um eine öffentliche Repräsentation sowie Aneignung, Vermittlung und Transformation vor allem geistlicher Wissensbestände geht. ${ }^{28}$ In diesem Kontext der Wissensvermittlung sind auch die im Meistergesang überlieferten Erzählungen und Geschichten über Lucifer zu deuten.

\section{Lucifer im Meistergesang}

Allein ein Blick ins Stichwortregister des ,Repertoriums der Sangsprüche und Meisterlieder' (RSM) zeigt, in welch vielfältiger Weise im Meistergesang mit den unterschiedlichen Bezeichnungen und Namen gespielt wird. Ein Blick auf die Stichwörter ,Satan', ,Teufel` und ,Lucifer‘ zeigt, dass die Nutzung des jeweiligen Stichwortes im Hinblick auf den jeweiligen Kontext nicht synonym zu erfolgen scheint. ${ }^{29}$ Der Begriff ,Teufel' findet am häufigsten Verwendung, weil er sich nicht nur auf den einen Teufel, den Höllenherrscher bezieht, sondern - in Einklang mit der Bedeutung des lateinischen Begriffs diabolos, die dem des hebräischen śâtān entspricht, auch für eine ganze Reihe dämonischer Geister und Höllenbewohner Verwendung findet. Der Begriff des oder der Teufel findet in der Regel in der Funktion des Verführers oder Heimsuchers Verwendung oder bei der Beschreibung unterschiedlicher Erscheinungsformen des Teufels. ${ }^{30}$ Der Großteil der Lieder hat narrativen Charakter und erzählt in Form von Exempla von den Taten des Teufels. ${ }^{31}$

Die Bezeichnung ,Satan' hingegen wird in erster Linie bei allegorischen Ausdeutungen und in Tiervergleichen verwendet. ${ }^{32}$ Darüber hinaus findet sich der

28 Vgl. Prautzsch, der auf Erich Kleinschmidt: „Literatur und städtische Gemeinschaft. Aspekte einer literarischen Stadtkultur in der Frühen Neuzeit“. In: Literatur in der Stadt. Bedingungen und Beispiele städtischer Literatur des 15. bis 17. Jahrhunderts. Hg. von Horst Brunner. Göppingen 1982, S. $73-93$, hier S. $77-78$ verweist.

29 Vgl. zum Stichwort ,Teufel‘ RSM 15, S. 564-567; zu ,Satan‘ S. 501-503; zu ,Luzifer‘ S. 388-389.

30 So erscheint der Teufel etwa als Bettler ( $\left.{ }^{2} \mathrm{Wat} / 113\right)$, als Mönch ( $\left.{ }^{2} \mathrm{Dei} / 176\right)$, als Pfaffe ( $\left.{ }^{2} \mathrm{Spr} / 133\right)$ oder als Ritter ( ${ }^{2}$ Met/308).

31 Es finden sich im RSM 15, S. 565f. bspw. die Stichpunkte: „Adliger wegen Fluchens und Spielens vom [Teufel] heimgesucht“ ( $\left.{ }^{2} \mathrm{HaG} / 311\right)$; „Als [Teufel] verkleidete Fastnachtsgäste erleiden tödliche Verbrennungen“ ( ${ }^{2}$ Dei/214); „Bleichergeselle wird vom [Teufel] gelähmt“ ( $\left.{ }^{2} \mathrm{HaG} / 310\right)$; „Leiche eines arianischen Bischofs von [Teufel]n weggetragen“ (2Wat/120); ,[Teufel] durch Furz vertrieben“ ( ${ }^{2} \mathrm{~S} / 499{ }^{2}$ Wat/251 254); „Ritter schwört vor einem [Teufel] Christus ab, doch nicht Maria“ ('Frau/23/3) oder „[Teufel] hält Furz einer Hexe für Seele“ ( $\left.{ }^{2} S / 2675\right)$.

32 So werden etwa Absalom ( $\left.{ }^{2} \mathrm{~S} / 3402\right)$, Amnon ( $\left.{ }^{2} \mathrm{~S} / 4675\right)$, Antiochus IV. ( $\left.{ }^{2} \mathrm{Hozm} / 1\right)$ oder Saul ( ${ }^{2} \mathrm{~S} /$ 582; $\left.{ }^{2} \mathrm{~S} / 1648 ;{ }^{2} \mathrm{~S} / 3715 ;{ }^{2} \mathrm{~S} / 3854\right)$ allegorisch auf den Satan gedeutet. Außerdem wird der Satan z. B. 
Begriff in Liedern, die sich mit den bösen Taten des Höllenfürsten beschäftigen. Dies erfolgt jedoch, anders als beim Begriff ,Teufel`, nicht primär anhand von Erzählungen über die Interaktion Satans mit einzelnen Menschen, sondern seine Taten werden allgemeiner angesprochen. ${ }^{33}$ Auch wenn die Lieder von der Verbindung zwischen Christus und dem ,Fürsten der Welt‘ oder Gottes Sieg über diesen handeln, wird der Begriff Satan dem allgemeineren Begriff ,Teufel' vorgezogen. Es scheint, als fände in den Meisterliedern der Ausdruck ,Satan` eher bei Bildungsinhalten Verwendung, während die volkstümlichen Exempla schlicht mit dem Begriff ,Teufel‘ auskommen. ${ }^{34}$

Der Fokus soll nun auf die Frage gerichtet werden, wann der dritte Teufelsbegriff, der Name ,Lucifer‘, im Meistergesang maßgeblich Verwendung findet. Auch hier erlaubt ein Blick ins Stichwortregister des RSM (Bd. 15) bereits erste Hypothesen: Mit Abstand die meisten Lieder, die den Namen Lucifer enthalten, befassen sich mit seiner Empörung wider Gott, seiner Vertreibung aus dem Himmel und seinem Sturz. Die Tatsache, dass hiervon wiederum die meisten Lieder im inhaltlich auf die Heilsgeschichte ausgerichteten ,Langen Ton' Regenbogens $^{35}$ verfasst sind, weist auf die besondere Stellung Lucifers in der Soteriologie hin. Indirekt umkreist wird dabei die Frage, warum Gott, bereits vor der Schöpfung von Lucifers Abkehr wissend, diesen erschuf.

Besonders die zahlreichen Johannesvisionen setzen sich in diesem Zusammenhang immer wieder mit der Unmöglichkeit des Nachvollzugs des göttlichen Handelns auseinander. Kein Mensch sei in der Lage, die göttlichen Ratschlüsse

mit dem Hamster ( ${ }^{W}$ Wat/399), dem Krokodil ( $\left.{ }^{2} \mathrm{WiH} / 19\right)$, dem Löwen $\left({ }^{2} \mathrm{Wat} / 180 ;{ }^{2} \mathrm{Wat} / 399\right)$ oder dem Luchs ('Wartb/2/1a+N1) verglichen.

33 Der Satan verursacht Krieg ( ${ }^{2}$ Wat/463/1), Melancholie ( ${ }^{2} \mathrm{Wat} / 186$; $\left.{ }^{2} \mathrm{Wat} / 406\right)$, Mord $\left({ }^{2} \mathrm{~A} / 1008\right.$; ${ }^{2}$ Wat/106) oder will das Reich Christi ( $\left.{ }^{2} \mathrm{Voi} / 164\right)$ bzw. das Werk Gottes $\left({ }^{2} \mathrm{~A} / 926\right)$ zerstören. Er wird als Feind der christlichen Lehre $\left({ }^{2} \mathrm{~S} / 4700\right)$, als Feind der Menschen $\left({ }^{2} \mathrm{~S} / 903\right)$ und als Fürst der Welt $\left({ }^{2} \mathrm{~S} /\right.$ 186) bezeichnet. Andererseits aber wird davon berichtet, dass das Wort Gottes den Satan besiegt $\left({ }^{2}\right.$ Voi/103), vor dem Satan bewahrt $\left({ }^{2} \mathrm{~S} / 3402\right.$; ${ }^{2} \mathrm{Wat} / 279$; 677) oder im Kampf gegen Satan hilft ( ${ }^{2}$ Faulh/2).

34 Diese, bisher nur anhand des Stichwort-Registers gezogenen Schlüsse bedürfen freilich einer tiefergehenden Untersuchung, die an dieser Stelle nicht weiterverfolgt werden soll. Als ebenfalls weiter erforschenswert erscheint die auffällige Häufung der Erwähnung Satans in Liedern des Hans Sachs.

35 Eine Analyse der geistlichen Lieder im ,Langen Ton` Regenbogens zeigt (besonders in der ,Kolmarer Liederhandschrift') eine deutliche Häufung heilsgeschichtlich-spekulativer Themen auf; breit vertreten sind Spekulationen über das Wesen der Trinität, Inkarnationsberichte sowie Johannesvisionen. Frieder Schanze (S. 70) sieht darin einen klaren Ausdruck von „Geltung und Rang des Tons“. Dass diese Fokussierung bereits im dreizehnten Jahrhundert zur Kenntnis genommen wurde, zeigt ein polemisches Zitat Heinrichs von Mügeln: In siner langen wise von des himels ort / spricht Regenbogen. daran sin tichten stet vermort. Zitiert nach Stackmann, Nr. 3, $1 \mathrm{f}$. 
mit seinem Verstand zu begreifen. Niemand könne bis zum Grund der göttlichen Absichten vordringen. Dieses Grundverständnis vom göttlichen Wesen ist ein zentrales Thema des Meistergesangs. Es finden sich unzählige Lieder, die um die Unergründlichkeit Gottes kreisen oder sogar davor warnen, sie durchgründen oder durchsinnen zu wollen. Als Preis für zu tiefes Schürfen nach dem göttlichen Wesen wird nicht selten der Verlust des Verstandes benannt. So heißt es in der zweiten Strophe des Liedes ${ }^{1} \operatorname{Regb} / 4 / 516:{ }^{36}$

II

Ach mensch, du solt nit wunder haben, wer und wo, waß got in sinem wesen sy und wo er hab gewonet vor, ee daz er habe hymmel und erd beschaffen, mit synnen nit zu ferre graben;

er ist eyn ungemessen got, kunst wont ym by, $\mathrm{du}$ wirst in dynen synnen ein tor.

[...]

wer weiss den endelosen grunt, syn hohe majestat und wie es got alz selb geformet hat mit meinsterlichen hant? der krafft mag an got nymant widerstan. der fal wart Lucifer bekant: ach unde we, daz muss er ymmer han mit allen den genossen sin.

umb die gedenck, die sie da hetten tratt, sie wurden in den tott verwuint und musten scheyden uff der helle pfat.

Geschickt wird die Warnung vor allzu starkem Ringen um Erkenntnis mit dem Fall Lucifers verknüpft, der als mahnendes Beispiel für Ungehorsam angeführt wird. Der Einzige, dem tiefere Einsicht gewährt wird, ist der auf Christi Brust in Schlaf versunkene Evangelist Johannes. Die auf der Apokalypse des Johannes fußenden Johannesvisionen bilden im Meistergesang das Mittel der Wahl, um im Grunde unbegreifliches Wissen über Gott, den Aufbau des Himmels sowie die Zusam-

\footnotetext{
36 Alle Lieder und anzitierten Liedstrophen in Regenbogens ,Langem Ton` (RSM Nrn. ${ }^{1}$ Regb/4/ 516, ${ }^{1} \operatorname{Regb} / 4 / 550,{ }^{1} \operatorname{Regb} / 4 / 632$ und $\left.{ }^{1} \operatorname{Regb} / 4 / 666\right)$ werden im Rahmen des von der DFG geförderten Projekts „Edition der in Regenbogens ,Langem Ton“ überlieferten Lieder unter der Leitung von Martin Schubert (Universität Duisburg-Essen) vollständig ediert und sind nach dieser im Entstehen begriffenen Ausgabe zitiert. Eine Ausnahme bilden die Zitate aus ${ }^{1}$ Regb/4/548, diese sind der bereits vorliegenden Edition von Wunderle, Nr. 12, entnommen.
} 
menhänge der Heilsgeschichte zu vermitteln. Gerade die neun Engelschöre sowie die Topografie von Himmel und Hölle bilden ein immer wiederkehrendes Thema in der frühen meisterlichen Liedkunst und im späteren Meistergesang. Hier zeigt sich der nachhaltige Einfluss Dionysius (Pseudo-)Areopagitas, ${ }^{37}$ dessen um 530 n. Chr. aufgestellte Angelologie auf das gesamte Mittelalter und die Frühe Neuzeit maßgeblich wirkte. ${ }^{38}$ Wie Kurt Ruh festhält, prägt Dionysius die Vorstellung „vom Prinzip der Hierarchie, die nicht nur ein theologisches Ordnungssystem, sondern ein spirituelles Modell der Vervollkommnung mit dem Ziel des ,Ähnlich- und Einswerdens“ mit Gott darstellt“. ${ }^{39}$ Gott kommt in diesem Ordnungssystem die Stellung des über allem thronenden, unergründlichen und unmessbaren Schöpfers zu, der den Anfang einer Kette bildet, ohne ein Glied derselben zu sein. Die obersten Glieder dieser Kette sind die neun Chöre der Engel, die wiederum in drei Hierarchien unterteilt sind. Eine Sonderstellung innerhalb des Systems kommt der ersten Triade - bestehend aus Thronen, Cherubim und Seraphim - zu, denn diese erhalte ihre Kräfte, nämlich Reinheit, Erleuchtung und Vollendung, direkt von Gott, ${ }^{40}$ um den sie unmittelbar kreisen:

sie [die Engel der ersten Triade, Anm. JL] sind nicht so aufzufassen, als ob sie gegenständliche oder begriffliche Symbole schauten oder durch die bunte Vielfalt der durch geheiligte Textbilder vermittelten Anschauung hindurch zum Wesen Gottes emporgeleitet würden, sondern in dem Sinn, daß ein Licht sie erfüllt, das noch über aller materiefreien Erkenntnis steht, und sie sich nach ihrer Bestimmung an dem das Sein übersteigenden, in dreierlei Form erscheinenden Anblick der Schönheit schaffenden Urschönheit sättigen, weiter, daß sie im gleichen Sinn der Gemeinschaft Jesus gewürdigt sind nicht in Bildern der heiligen Formensprache, die gestalthaft die göttliche wirkenden Angleichung (an Gott) abbilden, sondern so, daß sie sich ihm in voller Wahrheit nähern in der ersten (unvermittelten) Teilhabe an der Erkenntnis der von ihm ausgehenden Lichtstrahlen göttlicher Wirksamkeit, und weil sie in der Tat mit der Gabe der nachahmenden Darstellung Gottes im höchsten Grade beschenkt worden sind und weil sie in dem ihnen möglichen Grad in der in ihnen erstmals wirksam gewordenen Kraft an seinen göttlich wirkenden, menschenfreundlichen Vorzügen teilhaben. ${ }^{41}$

37 Vgl. zum Status Dionysius’ als ,falscher‘ Aposteljünger Ruh, S. 31.

38 Dionysius' Einteilung wurde (mit geringfügigen Abweichungen) in den nachfolgenden Jahrhunderten nahezu universell übernommen. Kommentare zu Dionysius finden sich u. a. in Hugos von St. Viktor In Hierarchiam Celestem, Albertus’ Magnus Super Dionysii Mysticam Theologiam oder Thomas' von Aquin Summa theologica.

39 Ruh, S. 43.

40 Vgl. Ruh, S. 56.

41 Pseudo-Dionysius, S. 45. 
Dionysius’ Beschreibung der Engel, besonders der ersten Triade, ist geprägt vom Schwanken zwischen intimer Nähe der höchsten Geschöpfe zu Gott bei gleichzeitig bestehender unermesslicher und unüberwindbarer Distanz. Die Engel werden zum einen als ewig seiend beschrieben und undifferenziert mit Gott zusammengeschlossen. Zum anderen, so Bernhard Brons, liegt die „ontologische Differenz der Engel zu Gott [...] auf dem Verursacht- bzw. Geschaffensein der Engel. [...] Die Engel sind darum in ihrem Sein Gott nachgeordnet, in ihrem Leben und in ihrer Erkenntnis stehen sie in einem Abbildverhältnis zu Gott“42 Dieses Changieren zwischen Nähe und Distanz, zwischen Schaffen und Geschaffensein bekundet sich in Johannes' passiver Haltung des Schauens besonders intensiv. Die Empörung und der Sturz Lucifers und seiner als genossen bezeichneten Anhänger (so auch in Frau/6/504) gehören dabei zum festen Repertoire der Visionsberichte. So heißt es exemplarisch in Strophe III des Liedes ${ }^{1} \operatorname{Regb} / 4 / 550$ :

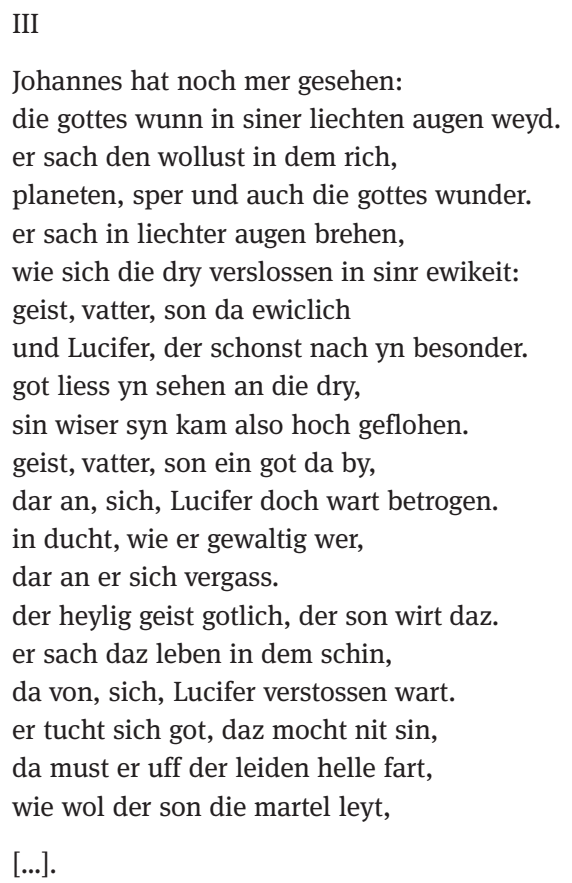

[...].

In nahezu allen Visionstexten zeigt sich eine Beschreibung Lucifers, die, wie gezeigt, bei Origenes ihre Entsprechung findet: Lucifer handelt selbstständig und ist wesensmäßig böse; gleichzeitig aber ist sein Sturz von Gott vorhergesehen und

42 Brons, S. $43 \mathrm{f}$. 
Teil des göttlichen Heilsplans. Besonders deutlich werden diese Zusammenhänge in der Strophe III des Liedes ${ }^{1}$ Regb/4/666:

III

Das hat ain >a alles gepaue, und auch ain »l das hat gestifftet grosses mort;

davan Isaias vil seit.

ich sorg, der fal der wert schwärlich gerochen.

jedoch het es got nit geraue,

wie wol gros not gestifftet het Adam das wort.

darum das `k hat ewig leit,

und auch das >a< das het schwerlich geprochen.

$\mathrm{a}, \mathrm{v}$ und e das wider pring

und was, das het das erste a verschulte.

da got al nach der menscheit ring,

dar umb das ıa dem $>$ k sich het verhulte.

das $\gg$ k das ist der Luzifer,

der hat gestifftet nat,

und da Adam zerprach, was im got pat.

got vatter in dem oberlant

der het an si geleget seinen fleis.

dem sun dem det der schaden ant,

und der geschach wol in dem paradeis.

es was die schult des Sathanas,

da Eva folget seinem pessen rat.

darum des sunes menscheit her

wol vir die schult muest leiden hie den dat.

Im Aufgesang - ebenfalls mit Berufung auf die Apokalypse des Johannes - ist der Zusammenhang zwischen dem Fall Lucifers (angedeutet durch den Verweis auf Jesaja), den durch Lucifer verursachten Sündenfall und Marias Bedeutung als ,neue Eva' für die Erlösung (III,9: $a, v$ und e das wider pring) im Buchstabenspiel deutlich nachvollzogen. Der Sturz Lucifers bildet den Anfangspunkt einer Kette von Ereignissen, die den Gnadenakt Christi bedingen. Im Abgesang wird nochmals die Schuld des Teufels am Sündenfall und seine ursächliche Schuld an der Marter Christi, der vir die schult muest leiden hie den dat, betont.

Über die Tatsache hinaus, dass sich Lucifer als gefallener Engel gut in den Heilsplan Gottes integrieren lässt und somit zum Werkzeug des Herrn wird, ermöglicht die Figur eine theoretische Gleichsetzung des gefallenen Engels mit dem sündigen Menschen. Die Lucifer traditionell zugeschriebene Sünde ist die Ursünde der superbia, d.h. die mittelhochdeutsche hoffart, zu der sich in der Regel Neid (sowohl auf Gott als auch auf den neu erschaffenen Menschen) gesellt. Das Schicksal Lucifers vermag, flankiert vom Schicksal Adams und Evas, der Er- 
mahnung aller Menschen zu dienen, sich vor hoffart in Acht zu nehmen, und zu gottesfürchtigem Leben aufzurufen. Gerade diese Form der Ermahnung und Belehrung findet in der meisterlichen Liedkunst und im Meistergesang ihren Niederschlag. Heinrich von Meißen etwa warnt vor zwifel und verwendet hierfür Lucifer als Exempel - die Edlen werden, ebenfalls vor dem Hintergrund der Lucifer-Geschichte, zu triuwe aufgerufen (vgl. ${ }^{1}$ Frau/2/112). Ein Anonymus dichtet in Frauenlobs ,Grünem Ton' ein Scheltlied wider die Hoffart ( $\left.{ }^{1} \mathrm{Frau} / 4 / 517\right)$. Wie schon Lucifer zuvor stürze die Hoffart auch heute noch manchen Sünder in die Hölle:

der tufel wart verstossen, da ist die hoffart schuldig an hoffart noch mangem schaden tut.

ubermut in zu der helle bringet.

wilt du nit abe lassen die wyl dir got die zyt hie gan

Lucifers wirst genossen, wiltu der hoffart nit enlan. (München, BSB, Cgm 4997, fol. 221 v)

Die Verbindung der Todsünden mit dem Namen und Wesen Lucifers macht den Teufel in der Gestalt des gefallenen Engels für die Meistersinger auch poetologisch zu einem fruchtbaren Thema. In den poetologisch-reflexiven Gattungen ,Fürwurf‘ und ,Straflied' erscheint Lucifer neben seiner Funktion als Exempel für alle Menschen auch speziell als mahnendes Beispiel für den hoffärtigen Sänger, sich und die eigene Kunst nicht zu überschätzen. ${ }^{43}$ So warnt das lyrische Ich in der ersten Strophe des Liedes ${ }^{1} \mathrm{Regb} / 4 / 632$ seinen Konkurrenten: Singer, ich rat dir auf mein trewe, / geleich mich niendert zů furwar. | [...] | es mecht dich wenge wol gerewe, I das schaffet als dein hoffart und dein ubermüt (I,1-6). Täte er dies doch, geschähe ihm, was Lucifer geschah, der sich in seinem Hochmut gottgleich wähnte und daher ungemach erleiden musste:

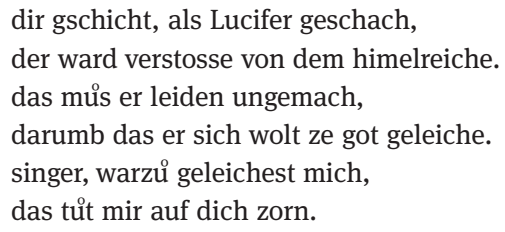

Der sich aus diesem Bild gleichfalls ergebende Vergleich des Sänger-Ichs mit Gott ist allenfalls insofern intendiert, als dass Gott im Meistergesang regelmäßig als der erste Meister und Kunstschaffende bezeichnet wird und die fähigen Sänger ihre Kunstfertigkeit in erster Linie von Gott erlangen. ${ }^{44}$ Es verwundert daher nicht,

43 Vgl. zu den Gattungen ,Fürwurf‘ und ,Straflied“ besonders Lange, S. 119-121.

44 Vgl. ebd., S. $116 \mathrm{f}$. 
dass die Strophe mit einer Anrufung Gottvaters und Christi endet, die Unfähigkeit des Konkurrenten wahrzunehmen und diese zu ahnden. Die Möglichkeit einer Bestrafung durch Gott wird wiederum in einer erneuten, mahnenden Anrede an den Konkurrenten selbst untergebracht:

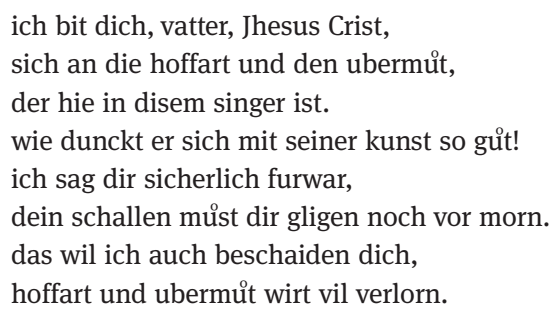

Mit seinem exponierten Verweis auf die Konsequenzen einer Hingebung an die Hoffart und einer Neigung zu übermuot (,hochfahrender, stolzer Sinn') im letzten Vers der Strophe richtet sich das Ich zwar in erster Linie an den fiktiven ermahnten Konkurrenten, darüber hinaus ist der Strophenschluss aber wohl auch als generelle Ermahnung an alle RezipientInnen zu lesen. Das dich (I,22) muss sich nicht mehr ausschließlich auf den Konkurrenten beziehen, sondern vermag das Publikum gleichsam mit einzuschließen. Poetologische Ausführungen werden folglich mit einer für das geistliche Lied typischen allgemeingültigen Didaxe verbunden, und die Thematik wird somit generalisiert.

Besonders geschickt verknüpft findet sich das Moment der Belehrung mit den Themen Wesen Gottes bzw. Trinität, Schöpfungsbericht inklusive Erschaffung des Himmels und der Erde sowie Lucifers Sturz aus dem Himmel in einem anonymen Meisterlied mit der Überschrift VII lieder von der geschopfft und von dem val der engel, welches in der ,Kolmarer Liederhandschrift' (München, BSB, Cgm 4997, fol. $377^{\mathrm{r}}-378^{\mathrm{r}}$ ) und in Heidelberg, UB, Cpg 680, fol. $18^{\mathrm{r}}-19^{\mathrm{v}}$ überliefert ist ( ${ }^{1} \mathrm{Regb} / 4 /$ 548). ${ }^{45}$ Anders als die zahlreichen Johannesvisionen weist das Lied starke narrative Strukturen auf.

Die erste Strophe berichtet in zum Teil verdunkelter Form vom Wesen der göttlichen Trinität, vom Schöpfungsbeschluss der Dreifaltigkeit. Die Verse 13-20 beschreiben den Aufbau des göttlichen Aufenthaltsortes (wesen, I,5;10), der von Gott als Min kor (I,22; ,meine [Himmels]-Chöre') bezeichnet wird und sich aus dem Firmament und den Himmelssphären zusammensetzt. Anschließend folgt in der zweiten Strophe ein durchaus typisch zu nennender Bericht über die Schöpfung der himmlischen Chöre, in deren höchstem Gott selbst seinen Wohnort haben wollte, und über die

45 Der nach München, BSB, Cgm 4997 edierte Text findet sich mit Kommentar bei Wunderle, Nr. 12. 
Erschaffung der Engel. ${ }^{46}$ In diesem Zusammenhang wird davon erzählt, dass Gott einen Engel erschuf, der trug aller engel cron (II,6) und der sich durch besondere Schönheit auszeichnete: er was durch lucht mit wunniclicher schone (II,8). Es folgt in den anschließenden Versen ein Bericht über den Abfall Lucifers von Gott aufgrund seiner Hoffart; in seinem Tun geleitet wird der Engel von dem Eindruck, als Gottes Abbild auch der Macht nach gottgleich zu sein (II,13f.: er glichet sich dem schopfer sin, mit siner schone gar $^{47}$; und II,20 f.: Lucifer blickt den schopfer an und sach sich selb in der gotheite clar).

Lucifer, das höchste Wesen der Schöpfung, vermag die Distanz zu Gott nicht zu überbrücken und strebt hier wohl gerade deswegen nach absoluter Freiheit, die jedoch per se nur Gott selbst, nicht aber den geschaffenen Wesen, zukommt. Den von Gott erschaffenen Himmel empfindet er als zu klein für sich und Gott und äußert den Wunsch: diss wesen dorfft ich wol alleine. (II,12). Interessant ist Gottes Antwort auf Lucifers Wunsch, Herrscher über den Himmel zu sein: nu mach dir selb ein zesen, | wie du daz vor dir hast in dinem mut! / dar inn solt ewiclichen wesen. / daz mach dir, wie du wilt, boß oder gut (II,16-19). Die Entstehung der Hölle als Ort des Schreckens, der Verdammung und der Bestrafung wird an dieser Stelle als Entscheidung Lucifers imaginiert, die zwar von Gott abgesegnet ist, nicht aber als eigentlich göttliche Entscheidung, ein ,böses‘ Pendant zum Himmel zu erschaffen, gelten kann. Üblicherweise bleibt die Frage, wer die Hölle erschuf, in den scholastischen Schriften der Kirchenväter sowie in der mittelalterlichen erzählenden Literatur ausgeklammert. ${ }^{48}$ Auch im Meistergesang finden sich keine Hinweise auf die Hölle als Schöpfung Gottes, es wird immer nur von der Erschaffung des Himmels und der Sphären gesprochen. Die Hölle wird auffällig aus dem Weltenaufbau ausgeklammert, obwohl deren Existenz als Ort der Bestrafung und des Leides sowie seine soteriologische Bedeutung (Verbannung Adams und Evas in die Hölle, Aufsprengen der Höllenpforte durch Christus und Maria) zum festen Repertoire des vermittelten Wissens im Meistergesang gehören. ${ }^{49}$

46 Sehr Ähnliches findet sich u. a. bei Michel Beheim.

47 Ähnliche Formulierungen finden sich im Lied noch häufiger: Der hymmel mocht hoffart nit tragen, / da wolt ein engel sinem schopfer wesen glich. / da von er uss dem hymmel quam, / da got geschaffen hett der engel bilde (V,1-5).

48 Die überzeitliche Existenz der Hölle wird vielmehr als gegeben anerkannt und lediglich über den ,Nutzen' derselben debattiert. Dabei stehen sich die durch Origenes geprägte Lehre der Apokatastasis und die maßgeblich durch Augustinus vertretene Vorstellung der Unendlichkeit der äonischen Höllenqualen ohne Hoffnung auf Erlösung nahezu diametral gegenüber. Vgl. hierzu Lang, S. 56-58. Dinzelbachers (S. 62) vereinfachende Aussage, „geschaffen hat sie [d.i. die Hölle] Gott für die abgefallenen Engel, die dort als Teufel hausen und die Verdammten bestrafen“, kann zumindest nicht anhand mittelalterlicher scholastischer Quellen oder der Bibel belegt werden. 49 Das Bild zweier paralleler, zwischen Himmel und Erde befindlicher zesen (eines Gottes und eines des Teufels) wird verschiedentlich auch in anderen Meisterliedern sowie im Lucidarius 
Die hier vorgestellte Strophe bildet (soweit ich das zum jetzigen Zeitpunkt überblicke) die einzige Ausnahme. Das Lied entspricht der von Flasch angesprochenen moralischen Aufwertung Gottes. Nicht der ausnahmslos gute Gott selbst erschafft die Hölle, sondern er übergibt diese Aufgabe an Lucifer. Zwar erfolgt der Höllenbau mit der Erlaubnis Gottes, aber kreiert hat dieser den Höllenort nicht. Die Hölle ist hier in erster Linie als Spiegelbildnis der Verderbtheit Lucifers zu sehen. Der Engel erfindet einen Ort, der seinem Wesen entspricht - aber nicht, um selbst darin zu herrschen. Lucifer gibt sich auch nicht damit zufrieden, neben Gottvater in einem ,zweiten“ Himmel zu herrschen: Er will den Höchsten hinab ins Elend stürzen und bringt sich damit selbst zu Fall.

Die Entscheidung für den Bau eines bösen und gegen den Bau eines guten zesen (,Firmament‘ oder ,Herrlichkeit‘, gemeint ist wohl der gesamte Himmelsaufbau einschließlich der neun Engelschöre als Wohnsitz Gottes) wird in den letzten zwei Versen der zweiten Strophe durch eine Lichtmetapher verdeutlicht. Gottes lichter schin wart im ein pin. er und die sinen wurden missevar (II,22f.). Die besondere Schwere der Schuld zeigt sich darin, dass Lucifer, der Lichtträger, zur Bestrafung nicht nur in die Hölle verbannt wird und seinen eigenen Glanz und seine Schönheit verliert, sondern fortan generell nicht mehr in der Lage ist, helles Licht zu ertragen. Die Bestrafung mit Lichtscheu und die damit einhergehende Verbannung in die Dunkelheit ist Signal für die Endgültigkeit der Verbannung. ${ }^{50}$ Lucifer vermag nie wieder in den Himmel und zu Gott zurückzukehren, dessen Leuchten ihm auf ewig Schmerzen bereiten muss. Der äußeren und inneren Hässlichkeit des gefallenen Engels entspricht die Feindseligkeit des Lebensraums, den er kreiert und in dem er zur Strafe seines Hochmuts ewig wohnen muss. Die verwunderliche Tatsache, dass der Teufel eine schreckliche Hölle erschafft, obwohl er eigentlich wie im Himmel leben will, wird nun im Folgenden mit einer geplanten, aber gescheiterten List Lucifers erklärt. Nicht ahnend, dass Gott allwissend ist, plant der Engel, Gottvater selbst mithilfe dreier Spiegelsteine in die Hölle zu locken, auf dass Gott im Höllenfeuer verbrenne. Obwohl im Lied selbst nicht weiter

verwendet. Siebert und mit ihm Wunderle (S. 354) übersetzen den Begriff nicht mit ,Himmel` (vgl. Baufeld, S. 258), sondern mit ,Palästen“ (vgl. Siebert: Himmels- und Erdkunde der Meistersänger, S. 230 und 235f., und ders.: Meistergesänge astronomischen Inhalts, S. 189 und 191).Vgl. auch die Diskussion zu zesem stM. ,Herrlichkeit‘ oder ,Firmament‘ im Wörterbuch zur Göttinger FrauenlobAusgabe, S. 495.

50 Der Bericht über die Verbannung eines Engels in vollständige Dunkelheit findet sich bereits in dem im frühen dritten Jh. v. Chr. entstandenen ersten Buch Henoch, das u. a. den Inhalt von Gen 6 geradezu fabulös ausschmückt: Nachdem die Sintflut beschlossen war, entsandte Gott den Erzengel Rafael, um Azazel (einen der Engel, die die Menschen auf Erden zu Gottlosigkeit und Eitelkeit verführten [vgl. 1 Hen 8,1-4]) in ein tiefes Loch zu werfen und dort für immer einzuschließen. „Laß ihn [d.i. Azazel] dort für immer wohnen und bedeck sein Antlitz, dass er kein Licht schaue!“ (1 Hen 10,5). 
darauf eingegangen wird, ist der Einsatz von spiegelnden Objekten für die List wohl Ausdruck der narzisstischen Persönlichkeit des Schönsten aller Geschöpfe Gottes. ${ }^{51}$ Luzifer versucht, Gott durch einen Spiegeltrug zu übertölpeln, indem er die Hölle durch die Reflexion auf den am Eingang angebrachten Spiegelsteinen als Himmel erscheinen lässt. Vom eigenen Wesen schließt der Engel auf Gott und muss mit der anmaßenden Annahme, Gott zu kennen und überlisten zu können, zwangsläufig scheitern. Dass es sich bei dieser Erzählung nicht um kanonisches Wissen, sondern um Fiktion handelt - zumindest ist mir eine solche Geschichte auch nicht aus der apokryphen Tradition bekannt -, wird vom Dichter selbst angemerkt (III,12). Zunächst wähnt sich Lucifer erfolgreich, wird dann aber vom trinitarisch wiederkehrenden Gott für seinen Frevel mit dem Entzug seiner Schönheit und der Verbannung in die Hölle bestraft. Erneut wird die Hoffart für Lucifers Sturz verantwortlich gemacht:

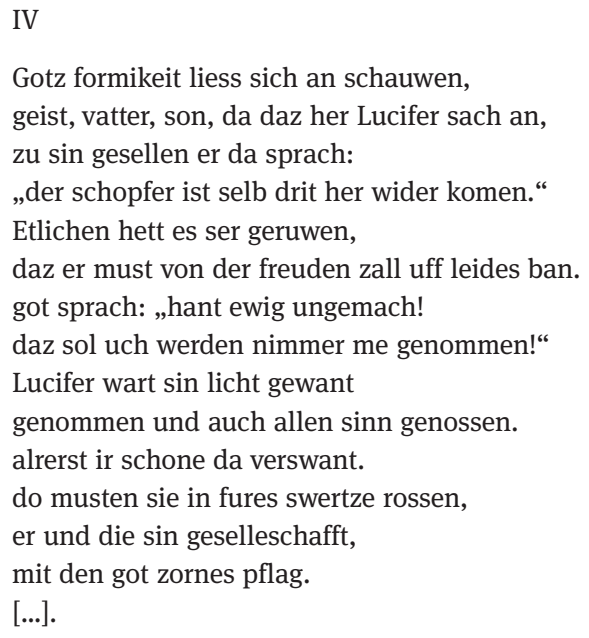

Die fünfte Strophe schlägt eine belehrende Brücke zur Schöpfung des Menschen. Diesem soll das Schicksal Lucifers als mahnendes Beispiel dienen. Gott selbst erklärt Adam und Eva, ihnen gebühre der von Lucifer und den Seinen geräumte

51 Besonders in der Märchenliteratur finden sich häufig Spiegel als Begleitobjekte hochmütiger Figuren oder sie dienen in Spiegelspielen einem betrügerischen Zweck. Die erste bildliche Kombination von Spiegel-Attribut und superbia findet sich im Welschen Gast Thomasins von Zerclaere und ab dem fünfzehnten Jahrhundert finden sich zunehmend Bildnisse, die die dezidiert weiblich personifizierte superbia mit Spiegel darstellen. Interessanterweise wird die superbia zunächst als hässliche Figur mit Spiegel dargestellt, erst im sechzehnten Jahrhundert wandelt sie sich zunehmend in eine schöne Frau. Vgl. Blöcker, S. 61 f., und zur grundsätzlichen Bedeutung von Spiegelspielen für das Er- und Verkennen Weber, S. 143-152. 
Platz in den himmlischen Chören, sofern sie dem Willen Gottes folgten (vgl. V,11-13). Vom Teufel mit einer List verführt, scheitern auch Adam und Eva und so müssen sie bis zum Jüngsten Tag auf Gottes Richtspruch warten (vgl. V,19f.). Das Lied endet mit dem Rückschluss auf den lebenden Menschen und einer Sündenmahnung: wer im [d.i. Gott] gebrist dann an der selben zal, der ist verloren $z u$ der stunt. hilff, herre got, in disem jamertal! (VII,21-23).

Die Figur des gefallenen Engels und seine Geschichte dienen auch hier schlussendlich als mahnendes Beispiel für den Menschen. Schlimmer noch als der Hoffart zu verfallen ist allein der Gedanke, man könne schlauer als Gott sein und ihn betrügen. Auch wenn die Strafe Gottes nicht auf dem Fuße erfolgt, bleibt doch keine Sünde von Gott unbemerkt. Einzig ein sündenfreies Leben kann zum ewigen Leben bei Gott führen; jede Zuwiderhandlung führt nach dem Tod in die Arme Lucifers und seiner Gefährten.

\section{Zusammenfassung}

Im gesamten Meistergesang finden sich keine Hinweise auf eine wie auch immer geartete Macht Lucifers. Seine Verbannung aus dem Himmel dient immer wieder als mahnendes Beispiel und gerade seine Ohnmacht gegenüber einem allmächtigen Gott wird zur Schau gestellt. Die Verbindung zwischen dem ,Fürsten der Welt' und den Menschen wird weitestgehend ausgeblendet; lediglich der Sündenfall der Menschheit, d.h. die Verführung Evas durch die Schlange, taucht an einer einzigen Stelle als Motiv in Verbindung mit dem Namen Lucifer auf. Die Vorstellung eines auf der Erde in unterschiedlichen Rollen agierenden Teufels oder - vermehrt unter der Bezeichnung ,Satan“ - die Vorstellung vom Verursacher unterschiedlichster Formen von Unheil und Schaden kommt im Meistergesang zwar vor, ist aber nie mit dem Namen Lucifer verknüpft. Die Funktion des gefallenen Lichtträgers bleibt vielmehr auf die Heilsgeschichte fokussiert. Starke Betonung findet Lucifers Rolle ganz traditionell in der Schöpfungsgeschichte präziser im Verlauf der Erschaffung von Himmel, Erde und Hölle. Zudem dient die Figur als Erklärungsmodell, wie sich das Böse mit der Allmacht und Schöpfung Gottes vereinbaren lässt: So wie Lucifer seinem Wesen nach ,böse‘ handelt, kann auch der Mensch durch sein selbstständiges Denken sündig handeln. Das von Neid und Hoffart durchdrungene Wesen Lucifers bietet sich in besonderem Maße als mahnendes Vorbild für den sündigen Menschen an. Wie auch Lucifer neigt der Mensch zu Hoffart und Neid, wodurch er sich immer in potentieller Gefahr einer Verbannung aus dem Himmelreich befindet. Besonders deutlich wird die Ähnlichkeit zwischen Lucifer und seinen Gefährten und der Menschheit im Lied ${ }^{1}$ Regb/4/666. Lucifer vermag, wie auch der Mensch, nicht, die Wesenheit Gottes 
vollends zu verstehen und verkennt dessen Allwissenheit. Daran scheitert sein listiger Betrugsversuch. Die Menschheit respektive Adam und Eva scheitern ebenso daran, den Geboten Gottes zu folgen, und auch sie scheint darauf zu hoffen, dass ihr Frevel nicht bemerkt würde. Dass Gott selbst der Menschheit in besagtem Gedicht zunächst den durch den Sturz Lucifers freigewordenen Platz im Himmel in Aussicht stellt - eine Aussage, die auch in anderen Liedern (z. B. ${ }^{1}$ Frau/ 6/105) getroffen wird - macht die Ähnlichkeit zwischen Lucifer und der Menschheit und die damit einhergehende Gefahr des Menschen für sich selbst umso augenfälliger.

\section{Literaturverzeichnis}

Baufeld, Christa: Kleines frühneuhochdeutsches Wörterbuch. Lexik aus Dichtung und Fachliteratur des Frühneuhochdeutschen. Tübingen 1996.

Blöcker, Susanne: Studien zur Ikonographie der sieben Todsünden in der niederländischen und deutschen Malerei und Graphik von 1450-1560. Münster, Hamburg 1993 (Bonner Studien zur Kunstgeschichte 8).

Brons, Bernhard: Gott und die Seienden. Untersuchungen zum Verhältnis von neuplatonischer Metaphysik und christlicher Tradition bei Dionysius Areopagita. Göttingen 1976.

Day, Peggy Lynne: An Adversary in Heaven. śāțān in the Hebrew Bible. Atlanta 1988.

Dinzelbacher, Peter: Jenseitsvisionen - Jenseitsreisen. In: Epische Stoffe des Mittelalters. Hg. von Volker Mertens, Ulrich Müller. Stuttgart 1984, S. 61-80.

Good, Edwin: Job and the Literary Task: A Response. In: Soundings 56 (1973), S. 470-484.

[Heinrich von Mügeln] Die kleineren Dichtungen Heinrichs von Mügeln. Hg. v. Karl Stackmann. 1. Abteilung: Die Spruchsammlung des Göttinger Cod. Philos. 21. 3 Teile. Berlin 1959 (DTM 50-52).

Lange, Judith: Wettstreit um die rehte kunst - Fürwurf und Straflied im Meistergesang des 15. Jahrhunderts. In: Die Edition mittelalterlicher deutscher Texte. Festkolloquium aus Anlass des 80. Geburtstags von Rudolf Bentzinger am 22.8.2016. Hg. von Eva Rothenberger, Martin Schubert, Elke Zinsmeister. Erfurt 2019, S. 113-137.

Lies, Lothar: Origenes ,Peri Archon“ - Eine undogmatische Dogmatik; Einführung und Erläuterung. Darmstadt 1992.

[Lutherbibel 2017] Die Bibel. Nach Martin Luthers Übersetzung. Lutherbibel, revidiert 2017. Jubiläumsausgabe 500 Jahre Reformation. Mit Sonderseiten zu Martin Luthers Wirken als Reformator und Bibelübersetzer. Hg. von der Evangelischen Kirche in Deutschland. Deutsche Bibelgesellschaft. Stuttgart 2016.

Martinek, Manuela: Wie die Schlange zum Teufel wurde: die Symbolik in der Paradiesgeschichte von der hebräischen Bibel bis zum Koran. Wiesbaden 1996.

Origenes: Vier Bücher von den Prinzipien. Hg., übersetzt, mit kritischen und erläuternden Anmerkungen versehen von Herwig Görgemanns, Heinrich Karpp. 3. Auf. Darmstadt 1992. Osterkamp, Ernst: Lucifer. Stationen eines Motivs. Berlin, New York 1979.

Pagels, Elaine: Satans Ursprung. Berlin 1996. 
Prautzsch, Felix: Die Meisterschaft des Laien? Zum ästhetischen und theologischen Anspruch im Meistergesang. Erscheint in: Die Kolmarer Liederhandschrift und ihr Umfeld. Hg. von Judith Lange, Eva Rothenberger, Martin Schubert.

Pseudo-Dionysius Areopagita: Über die himmlische Hierarchie. Über die kirchliche Hierarchie. Eingeleitet, übersetzt und mit Anmerkungen versehen von Günter Heil. Stuttgart 1986.

Rees, Valery: Von Gabriel bis Luzifer. Eine Kulturgeschichte der Engel. Darmstadt 2017.

Robertson, David: The Book of Job: A Literary Study. In: Soundings 56 (1973), S. 446-469.

Rosmer, Stefan: Die Tradition des geistlichen Sangspruchs und seine Gestalt um 1300. In: Sangspruchdichtung um 1300. Akten der Tagung in Basel vom 7. bis 9. November 2013. Hg. von Gert Hübner. Hildesheim 2015, S. 307-330.

Ruh, Kurt: Geschichte der abendländischen Mystik. Bd. I. Die Grundlegung durch die Kirchenväter und die Mönchstheologie des 12. Jahrhunderts. 2. Auflage. München 2001.

Schmidt, Joachim: Satanismus. Mythos und Wirklichkeit. Marburg 1992.

Siebert, Johannes: Himmels- und Erdkunde der Meistersänger. In: ZfdA 76 (1939), S. 222-252.

Siebert, Johannes: Meistergesänge astronomischen Inhalts. In: ZfdA 83 (1951/52), S. 181-235.

[Vulgata] Biblia Sacra Vulgata. Editio quinta. Hg. von Robert Weber, Roger Gryson. Stuttgart 2007.

Weber, Karin: Hochmut und Spiegelspiele. Über Spiegel, Hochmut und Erkenntnis in Jean Cocteaus Orphée. In: Superbia - Hochmut und Stolz in Kultur und Literatur. Hg. von Bozena Anna Badura, Tilmann F. Kreuzer. Gießen 2014, S. 143-182.

Wörterbuch zur Göttinger Frauenlob-Ausgabe. Unter Mitarbeit von Jens Haustein redigiert von Karl Stackmann. Göttingen 1990 (Abhandlungen der Akademie der Wissenschaften in Göttingen Philologisch-Historische Klasse 3, 186).

Wunderle, Elisabeth (Hg.): Studien zur Meisterliedüberlieferung. Edition des cpg 680 mit Kommentar. München 1988. 